\title{
A new water anomaly: the temperature dependence of the proton mean kinetic energy
}

\author{
D. Flamini, M. A. Ricci, and F. Bruni* \\ Dipartimento di Fisica "E. Amaldi", \\ Università degli Studi di Roma Tre, \\ Via della Vasca Navale 84, 00146 Roma, Italy
}

(Dated: December 3, 2018)

\begin{abstract}
The mean kinetic energy of protons in water is determined by Deep Inelastic Neutron Scattering experiments, performed above and below the temperature of maximum density and in the supercooled phase. The temperature dependence of this energy shows an anomalous behavior, as it occurs for many water properties. In particular two regions of maximum kinetic energy are identified: the first one, in the supercooled phase in the range $269 \mathrm{~K}-272 \mathrm{~K}$, and a second one above 273 K. In both these regions the measured proton kinetic energy exceedes the theoretical prediction based on a semi-classical model. Noteworthy, the proton mean kinetic energy has a maximum at $277 \mathrm{~K}$, the temperature of the maximum density of water. In the supercooled metastable phase the measured mean kinetic energy and the proton momentum distribution clearly indicate proton delocalization between two H-bonded oxygens.

PACS numbers: $61.25 . \mathrm{Em}, 78.30 . \mathrm{Cp}, 78.70 . \mathrm{Nx}$
\end{abstract}

[*] Author to whom correspondence should be addressed. E-mail: bruni@fis.uniroma3.it 
Among liquids, water is undoubtely the most studied, as it is the medium of life and chemistry on our planet, and its puzzling properties are a challenge for science [1, 2]. Yet water quantum behavior has received less attention than its classical thermodynamic properties, in spite of the modest cooling needed to evidence quantum effects in water. These have been recently suggested to determine the water density maximum [3] and shown to influence the molecular geometry [4] and in particular the $\mathrm{OH}$ bond-length. Quantum effects have also been evidenced in the short time dynamics of protons along the $\mathrm{OH}$ bond in supercooled [5] and confined water [6, 7, 8], by Deep Inelastic Neutron Scattering (DINS) [9] experiments and computer simulations [10, 11]. Here we report DINS measurements on bulk water in the stable phase around the density maximum and in the supercooled metastable regime, showing clear evidence for quantum effects on the momentum distribution $n(p)$ and mean kinetic energy $\left\langle E_{K}\right\rangle$ of water protons.

At present DINS is the only technique which determines the momentum distribution and mean kinetic energy of a single particle in condensed matter. It is based on neutron scattering measurements at high energy $(1 \mathrm{eV}<\hbar \omega<200 \mathrm{eV})$ and momentum $\left(120 \AA^{-1}<\hbar q<\right.$ $\left.300 \AA^{-1}\right)$ transfers, thus probing both the short-time dynamics and local environment of the particle. The high energy and wave vector transfers achieved allow the scattering process to be described within the framework of the Impulse Approximations (IA) [12]. This assumes that neutron scattering is incoherent and occurring within time scales much shorter than the typical relaxation times of the collective excitations of the system. In these conditions the struck particle recoils freely from the collision, with inter-particle interaction in the final state being negligible (i.e. the wave-function of the particle in its final state assumed to be a plane wave). In a molecular system, as for instance water, the contribution to the scattering cross section of protons can be easily distinguished from that of oxygens, due to the large mass difference. The scattering cross section is then expressed in terms of the single proton momentum distribution $n(p)$, whose variance is directely related to its mean kinetic energy $\left\langle E_{k}\right\rangle$. These quantities provide a richness of information about the potential surface that the proton experiences [13], including the effects of hydrogen bonding, thus complementing microscopic structural studies [4, 14, 15] and allowing a direct comparison with quantum Monte Carlo simulations [10, 11].

DINS experiments have been carried out on the VESUVIO spectrometer [9], at the ISIS spallation neutron source (UK). The samples were contained in the same disk shaped alu- 
minum can (5 cm diameter, $1 \mathrm{~mm}$ thickness) with inner Teflon coating used in previous work on supercooled water [5]. Experimental data have been recorded at 64 fixed-angle detectors in the standard Resonance Detector configuration [16] employing the Foil Cycling technique [17]. Data have been corrected for multiple scattering, aluminium and oxygen signal, by using the same routines as in previous work [5]. Within the IA framework the dynamical structure factor, $S_{I A}(\vec{q}, \omega)$, is related to the $n(p)$ through the relation:

$$
S_{I A}(\vec{q}, \omega)=\int n(\vec{p}) \delta\left(\omega-\frac{\hbar q^{2}}{2 M}-\frac{\vec{p} \cdot \vec{q}}{M}\right) d \vec{p}
$$

where $\hbar \omega$ is the energy transfer, $\frac{\hbar^{2} q^{2}}{2 M}$ is the recoil energy of the struck atom of mass $M$, and $q$ is the vavevector transfer. The standard deviation of the $n(p)$ function, $\sigma$, is related to $\left\langle E_{k}\right\rangle$, through the relation $\sigma^{2}=\frac{2 M}{3 h^{2}}\left\langle E_{k}\right\rangle$. The dynamical structure factor is then expressed in terms of a Neutron Compton Profile (NCP) [9]: $F(y)=\frac{\hbar q}{M} S_{I A}(\vec{q}, \omega)$, where $y=\frac{M}{\hbar q}\left(\omega-\frac{\hbar q^{2}}{2 M}\right)$ is the West scaling variable [9]. The NCP lineshape is convolved with the instrumental resolution function, and represented as a series expansion in Hermite polynomials:

$$
F(y)=\frac{e^{-\frac{y^{2}}{2 \sigma^{2}}}}{\sqrt{2 \pi} \sigma}\left[1+\sum_{n} \frac{c_{n}}{2^{2 n} n !} H_{2 n}\left(\frac{y}{\sqrt{2} \sigma}\right)\right]
$$

The coefficients $c_{n}$ and $\sigma$, appearing in the series expansion, have been determined by a least squares fitting procedure (see caption of Fig. 2), and small corrections due to deviations from the IA were taken into account [9]. We did not attempt to correct for the so-called intensity deficit seen for hydrogen relative to heavier nuclei [18, 19, 20]. Altough the issue of the intensity deficit in DINS experiment is still debated, it should be noted that in systems where the $n(p)$ and the NCP are purely gaussian, such as $\mathrm{ZrH}_{2}$, no distortion of the measured NCP is indeed observed [21]. Finally the $n(p)$ is expressed by the expansion [9]

$$
n(p)=\frac{e^{-\frac{p^{2}}{2 \sigma^{2}}}}{(\sqrt{2 \pi} \sigma)^{3}} \cdot \sum_{n} c_{n}(-1)^{n} L_{n}^{\frac{1}{2}}\left(\frac{p^{2}}{2 \sigma^{2}}\right)
$$

where $L_{n}^{\frac{1}{2}}$ are generalized Laguerre polynomials. Full details about data analysis may be found in ref. 9 .

Figure 1 shows the temperature dependence of proton's $\left\langle E_{k}\right\rangle$ measured in both stable and metastable phases of bulk water in the temperature range from $300 \mathrm{~K}$ to $269 \mathrm{~K}$, along with the semi-classical prediction, $E_{s c}$. The temperature dependence of $E_{s c}$ in the stable water 
phase, shown in Fig. 1 as a dotted line, has been derived in Ref. 22, taking into account translational, rotational, and vibrational contributions, including the optical spectroscopic data available in the literature. While data for ice [23] and water [16] above $293 \mathrm{~K}$, including supercritical states [22, 24], are satisfactorily described by the semi-classical prediction, data around the temperature of maximum density and in the supercooled phase show an excess of proton mean kinetic energy. In particular $\left\langle E_{K}\right\rangle$ shows two maxima: one at $277 \mathrm{~K}$ and the other in the supercooled phase in the range $269 \mathrm{~K}-271 \mathrm{~K}$, with an excess of $\left\langle E_{K}\right\rangle$ with respect to $E_{s c}$ of about $3 \mathrm{~kJ} / \mathrm{mol}(30 \mathrm{meV})$ and $12 \mathrm{~kJ} / \mathrm{mol}(120 \mathrm{meV})$, respectively. The peculiar temperature dependence of $\left\langle E_{k}\right\rangle$, suggests that two distinct mechanisms may be considered below and above the melting temperature. As a matter of fact the excess of $\left\langle E_{k}\right\rangle$ in the stable water phase is moderate and its temperature dependence follows that of density [25], showing a maximum at the same temperature (see insert in Fig. 1). A correlation between density and $\left\langle E_{k}\right\rangle$, as measured by DINS, is not a novelty in principle, as it has already been observed in helium [26, 27] and explained in that case by using a harmonic model for the fluid. Nevertheless the peculiar density behavior of water and the temperature evolution of the H-bond network does not allow a similar model to work in this case. We suggest instead that the anomaly of $\left\langle E_{k}\right\rangle$ vs $T$ above $273 \mathrm{~K}$ may be explained as a further evidence for water structural anomalies, which manifest through the existence of a maximum of density and transport properties in the stable water phase [28]. Within this hypothesis the maximum of $\left\langle E_{k}\right\rangle$ at $277.15 \mathrm{~K}$, shown in Fig. 1, may be an indirect manifestation of the competition between zero point energy, $E_{0}$, and thermal fluctuations, which has been proposed as the quantum origin of the density maximum [3]. We notice however that quantum effects are not necessarily required to explain the existence of a maximum of density in water [29].

On the other side the huge increase of $\left\langle E_{k}\right\rangle$ in the metastable states of water, already observed in a recent publication [5] and confirmed by present new measurements at 272.15 and $272.95 \mathrm{~K}$, seems to be directely related to the likely delocalization of protons along the H-bond. Fig. 2 shows the radial momentum distribution, $4 \pi p^{2} n(p)$, at 277.15 and 271.15 $K$. This function at the lowest temperature shows a narrowing at low- $p$ and a shoulder at high- $p\left(\sim 17 \AA^{-1}\right)$, compared to that measured at $277.15 \mathrm{~K}$, compatible with the transition from a single to a double well potential. The presence of a shoulder at high- $p$ in the radial momentum distribution has indeed been ascribed to coherent delocalization of protons over 
two sites of a double well potential [13] felt by the proton along the H-bond direction between two water molecules. In other words, a statistical ensamble of water molecules in which protons are localized in the vicinity of the covalently bonded oxygens is predicted to show a single maximum of $4 \pi p^{2} n(p)$ at $p \sim 6 \AA^{-1}$, corresponding to the intramolecular O-H distance $d=\frac{2 \pi}{p} \sim 1 \AA$, as shown in Fig. 2 and confirmed by quantum mechanical simulations [10, 11]. Conversely, the appearence of a shoulder at $p \sim 17 \AA^{-1}$ in the metastable supercooled phase indicates a delocalization of the proton over a distance $\Delta d=\frac{2 \pi}{\Delta p} \sim 0.6 \AA$ from the equilibrium position. We notice that $\left\langle E_{k}\right\rangle$ in the supercooled phase is comparable with the H-bond energy $(\sim 20 \mathrm{~kJ} / \mathrm{mol})$ and $\Delta d$ is ompatible with the width of the fluctuations of the H-bond length (width of the first intermolecular peak of the oxygen-hydrogen radial distribution function) and the oxygen-oxygen distance in supercooled water [14]. Based on this observation, we proposed [5] the quantum excess of proton mean kinetic energy be correlated to the average distance between two first neighboring H-bonded oxygens in water. Such assignement was corroborated by similar evidences in confined water [6, 8, 15]. Further evidences for a correlation between proton delocalization and distance between its neighboring oxygens are given in Ref. 30 and 31, showing the changes of the potential landscape, energy barrier and wave-function of a proton as a function of this O-O distance.

The present results show a clear and unexpected anomalous temperature dependence of the proton mean kinetic energy. Below $273 \mathrm{~K}$, in the supercooled phase, this anomalous behavior is associated with a coherent delocalization of the proton between first neighbor oxygens. Above $273 \mathrm{~K}$ the temperature dependence of the proton mean kinetic energy resembles that of water density.

The authors acknowledge useful discussion with C. Andreani, A. Pietropaolo, R. Senesi, and J. Mayers. We are grateful to M. Adams for helpful assistance and discussions during the experiment. This work has been performed within the Agreement No.01/9001 between STFC and CNR, concerning collaboration in scientific research at the spallation neutron source ISIS and with partial financial support of CNR.

[1] P.G. Debenedetti, J. Phys.: Condensed Matter 11, R1669 (2003).

[2] Special Issue Phys. Chem. Chem. Phys. 2 (2000). 
[3] F. A. Deeney, and J. P. O'Leary, Phys. Lett. A 372, 1551 (2008).

[4] A. K. Soper, and C. J. Benmore, Phys. Rev. Lett. 101, 065502 (2008).

[5] A. Pietropaolo, R. Senesi, C. Andreani, A. Botti, M. A. Ricci, and F. Bruni, Phys. Rev. Lett. 100, $127802(2008)$.

[6] V. Garbuio, C. Andreani, S. Imberti, A. Pietropaolo, G. F. Reiter, R. Senesi, and M. A. Ricci, J. Chem. Phys. 127, 154501 (2007).

[7] R. Senesi, A. Pietropaolo, A. Bocedi, S. E. Pagnotta, and F. Bruni, Phys. Rev. Lett. 98, 138102 (2007).

[8] S. E. Pagnotta, F. Bruni, R. Senesi, A. Pietropaolo, Biophysical Journal 2009, doi:10.1016/j.bpj.2008.10.062

[9] C. Andreani, D. Colognesi, J. Mayers, G. F.Reiter, and R. Senesi, Adv. Phys. 54, 377 (2005).

[10] J. A. Morrone, V. Srinivasan, D. Sebastiani, and R. Car, J. Chem. Phys. 126, 234504 (2007).

[11] J. A. Morrone, and R. Car, Phys. Rev. Lett. 101, 017801 (2008).

[12] G. B. West, Phys. Rep. 18, 263 (1975).

[13] G. F. Reiter, and R. Silver, Phys. Rev. Lett. 54, 1047, 1985.

[14] A. Botti, F. Bruni, M. A. Ricci, and A. K. Soper, J. Chem. Phys. 117, 6196 (2002).

[15] M. A. Ricci, F. Bruni, A. Giuliani, Faraday Discussion 141, 347 (2009).

[16] P. Pietropaolo, C. Andreani, A. Filabozzi, R. Senesi, G. Gorini, E. Perelli Cippo, M. Tardocchi, N. J. Rhode, and E. M. Schooneveld, JINST 1, P04001 (2006).

[17] E. M. Schooneveld, J. Mayers, N. J. Rhodes, P. Pietropaolo, C. Andreani, R. Senesi, G. Gorini, E. Perelli Cippo, M. Tardocchi, Rev. Sci. Inst. 77, 095103 (2006).

[18] C. A. Chatzimidriou-Dreismann, T. Abdul Redah, R. M. F. Streffer, and J. Mayers, Phys. Rev. Lett. 79, 2839 (1997).

[19] R. Senesi, D. Colognesi, A. Pietropaolo, T. Abdul Redah, Phys. Rev. B 72, 054119 (2005).

[20] G. Reiter and P. Platzman, Phys. Rev. B. 71, 054107 (2005).

[21] A. C. Evans, D. N. Timms, J. Mayers, and S. M. Bennington, Phys. Rev. B 53, 3023 (1996).

[22] C. Andreani, D. Colognesi, E. Degiorgi, and M. A. Ricci, J. Chem. Phys. 115, 11243 (2001).

[23] G. F.Reiter, J. C. Li, J. Mayers, T. Abdul-Redah, and P. Platzman, Braz. J. Phys. 34, 142-147 (2004).

[24] C. Pantalei, A. Pietropaolo, R. Senesi, S. Imberti, C. Andreani, J. Mayers, C. Burnham, and G. Reiter, Phys Rev. Lett. 100, 177801 (2008). 
[25] http://webbook.nist.gov/chemistry,

[26] U. Bafile, M. Zoppi, F. Barocchi, R. Magli, and J. Mayers, Phys. Rev. B 54, 11969 (1996).

[27] F. Albergamo, M. Nardone, and A. Filabozzi, Phys. Rev. B 56, 14614 (1997).

[28] J. R. Errington, and P. G. Debenedetti, Nature 409, 319, 2001.

[29] F. Paesani, S. Iuchi, and G. A. Voth, J. Chem Phys. 127, 074506 (2007).

[30] E. A. Hillenbrand, and S. Scheiner, J. Am. Chem. Soc. 106, 6266, (1984).

[31] D. Marx, Chem. Phys. Phys. Chem. 7, 1848 (2006). 


\section{Figure Captions}

- Figure 1: Water proton mean kinetic energy $\left\langle E_{K}\right\rangle$ as a function of temperature. Open symbols have been used for water in the stable phases, solid symbols for the metastable supercooled phase, namely: ice [23] at $269 \mathrm{~K}$ (square); liquid stable water (circles). Water data above $293 \mathrm{~K}$ are taken from Ref. 16, those below $272.15 \mathrm{~K}$ are from Ref.

5. Present data are reported for temperatures in the range $272.15-285.15 \mathrm{~K}$. The dotted line corresponds to the semi-classical prediction [22]. The error bars are derived from the least squares fitting procedure used to derive $\left\langle E_{K}\right\rangle$ from the differential cross sections (see references 6, 9). The solid and dashed lines are guides for the eye. In the insert $\left\langle E_{K}\right\rangle$ data in the stable liquid phase (circles and right axis) are reported in comparison with the density of water (line plus solid triangles and left axis) as a function of temperature.

- Figure 2: Spherically averaged momentum distribution of water protons at the point of maximum density (present experiment, $T=277.15 \mathrm{~K}$ ), (dotted line) and in the metastable supercooled phase at $T=271 \mathrm{~K}$ (solid line, from Ref. 5). Experimental uncertainties are less than $\pm 1 \%$. Values for the fitting parameters (see Eq. 2-3) are as follows: $c_{1}$ is set to zero by definition, the non-Gaussian coefficient, $c_{2}$, and $\sigma$ are $(0.443 \pm 0.008)$ and $(6.05 \pm 0.03) \AA^{-1}$ at $T=271 \mathrm{~K} ;(0.148 \pm 0.010)$ and $(5.13 \pm$ $0.02) \AA^{-1}$ at $T=277.15 \mathrm{~K}$; the coefficients $c_{n \geq 3}$ are found to be negligible. 


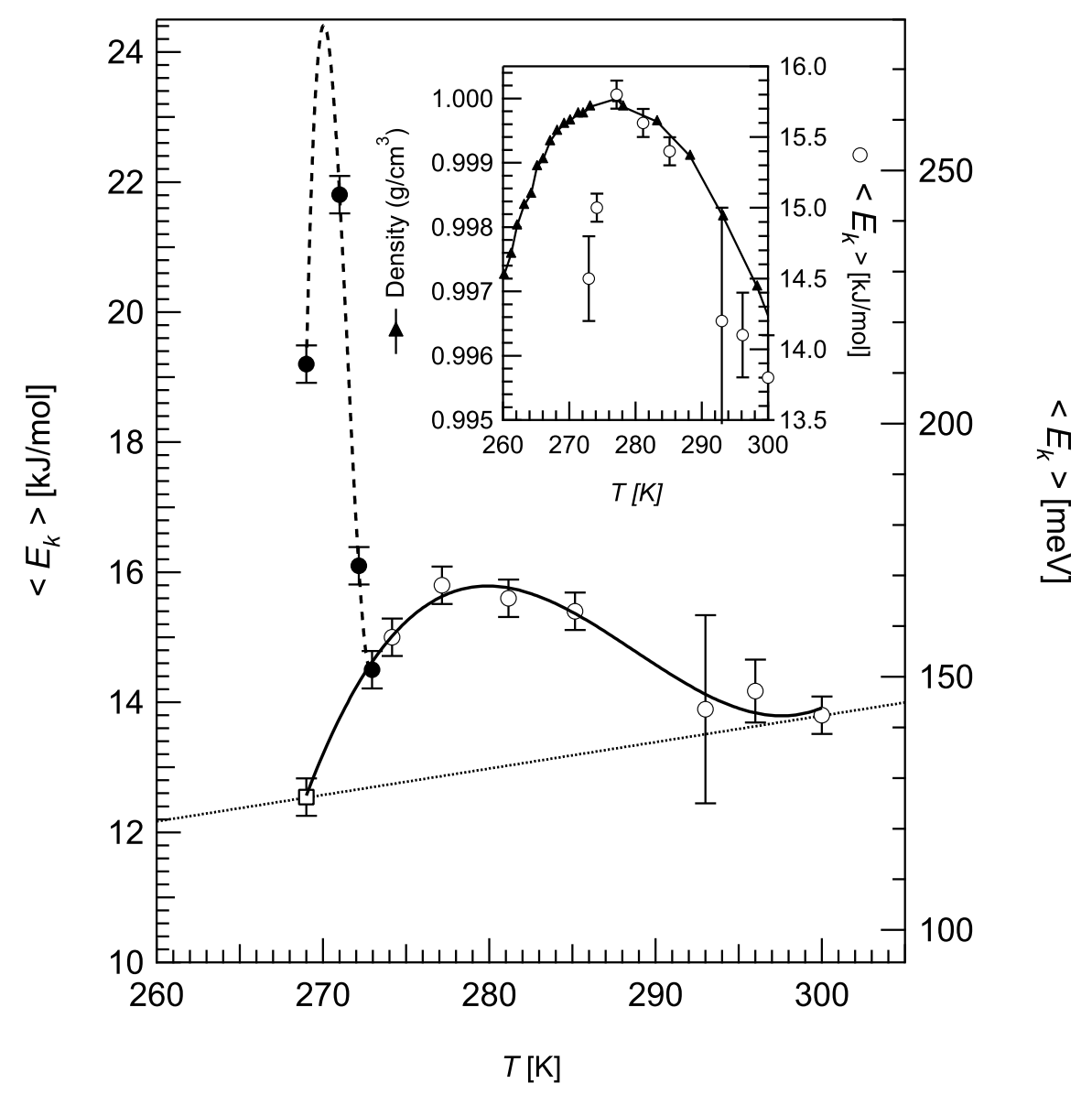

FIG. 1: 




FIG. 2: 